\title{
Orthopaedics for beginners
}

\section{It SHOULD be healed by now!}

\section{Eithne Comerford}

\section{INTRODUCTION}

The failure of a fracture to heal in the manner and timescale which is expected results in a delay of return to normal function or permanent disability. This is due to either an inadequate mechanical or biological environment around the fracture site.

\section{WHY HAS IT NOT HEALED?}

Inadequate mechanical environment will be associated with alignment, reduction of the fracture fragments, size of the fracture gap and the forces at the fracture site. An inadequate biological environment is one which the growth factors and bone cells are not supported to produce bone. Intrinsic (e.g. soft tissue attachments) and extrinsic (e.g. surgical technique) influences affect the fracture site envelope.

- Delayed union is a fracture has failed to heal in the time expected. It will either go on to heal or not (non-union). This is dependent on factors such as age of animal, which bone is fractured, the site of fracture and the type of implant used

- Non-union is when fracture healing has stopped. Non-unions may be broadly classified as those which are 'viable' and those which are 'non-viable'. Viable non-unions are further classified as hypertrophic, moderately hypertrophic or oligotrophic. Non-viable non-unions are described as dystrophic where there is a poor blood supply to bone fragments, necrotic where there is a major loss of blood supply to fragments, defect where a major gap exists between the fracture fragments and atrophic where there is a loss of blood supply to the whole fracture site causing an 'apple-core' appearance

- Infection/osteomyelitis is an inflammatory condition of bone usually caused by bacteria, fungi or viruses. Trauma, implant placement or direct inoculation can lead to osteomyelitis.

\section{HOW CAN I GET IT TO HEAL?}

One must first recognize the complication, identify factor(s) causing it and apply appropriate corrective treatment.

- Delayed and non-unions: be premptive to avoid these complications and plan in the postoperative period. For delayed unions, if the animal is weight-bearing, not infected and the fixation is stable then the cases should be closely monitored. In non-unions, implants and non-viable bone will need to be removed and biologics may need to be added to allow for bone regeneration. Understanding both the mechanical and biological environment around the fracture site will allow for an onward treatment plan

- Infection: antimicrobial therapy - intravenous for 3-5 days then oral for 4-6 weeks (often until fracture healed and implant can be removed if biofilm). For Staphylococcus spp. you would most commonly use first-generation cephalosporins or amoxicillin-clavulanate. Radiograph every 3-4 weeks to assess. Ensure that the fracture fixation/ implant present is stable to avoid compromised vascular supply. Aggressive treatment may be needed including drainage, debridement, direct bone/tissue culture and possibly delayed closure.

\section{KEY LEARNING OBJECTIVES}

- Identify when a fracture is not healing as expected by using common diagnostic tools such as clinical examination and imaging

- Critically evaluate the fracture fixation, categorize the stage of healing and identify any causative factors

- Develop an onward plan to manage the delayed fracture repair

\section{MULTIPLE CHOICE QUESTIONS}

1. Which of the following statements about delayed non-unions is correct?

(A) Have no blood supply at the fracture site

(B) Will never heal

(C) Need immediate aggressive treatment

(D) Should be closely monitored if stable

2. How are non-unions treated?

(A) With a long course of antibiotics

(B) Following assessment of the fracture site environment

(C) By leaving the non-viable bone fragments at the fracture site

(D) By placing autogenous cancellous bone graft at the fracture site

3. Diagnosis of an infected fracture is made most commonly by which of the following?
(A) Magnetic resonance imaging and computed tomography
(B) Clinical examination and radiography
(C) Fine needle aspirates of the fracture site
(D) Response to antibiotic therapy 